Z Gerontol Geriat 2017 · 50:262

DOI 10.1007/s00391-017-1209-3

Online publiziert: 6. März 2017

(c) Springer Medizin Verlag GmbH 2017

CrossMark

\section{Mehr Blutungskomplikationen unter Rivaroxaban}

\section{Originalpublikation}

Graham DJ et al (2016) Stroke bleeding, and mortality risks in elderly medicare beneficiaries treated with Dabigatran or Rivaroxaban for nonvalvular atrial fibrillation. JAMA Int Med 176(11):1662-1671

Rivaroxaban in einer Dosis von $20 \mathrm{mg} / \mathrm{Tag}$ ist im Vergleich mit Dabigatran 2-mal $150 \mathrm{mg} /$ Tag mit einem statistisch signifikanten Anstieg intrakranieller Blutungen und größerer extrakranieller Blutungen, einschließlich größerer gastrointestinaler Blutungen, verbunden. $\mathrm{Zu}$ diesem Ergebnis kommt eine retrospektive Kohortenstudie unter 118.891 Patienten. Die Patienten - alle älter als 64 Jahre - wurden wegen eines nichtvalvulären Vorhofflimmerns antikoaguliert. Die Untersuchung stützt sich auf MedicareDaten des Zeitraums von November 2011 bis Juni 2014. Bei der statistischen Auswertung wurden die unterschiedlichen klinischen Charakteristika der Patienten berücksichtigt. Unter Rivaroxaban wurden zwar weniger thrombembolische Schlaganfälle beobachtet, doch war dieser Unterschied statistisch nicht signifikant. Unter den Patienten jenseits des 75. Lebensjahres sowie unter Hochrisikopatienten (CHADS 2-Score >2) nicht jedoch in der Gesamtkohorte ging die Therapie mit Rivaroxaban im Vergleich zu Dabigatran mit einem statistisch signifikanten Anstieg der Mortalität einher.

\author{
R. Püllen \\ Agaplesion Frankfurter Diakonie-Kliniken, Frankfurt a. M., Deutschland
}

\title{
Journal Club
}

\section{Arterieller Blutdruck und Mortalität}

\section{Originalpublikation}

Morrisey Y et al (2016) Older people remain on blood pressure agents despite being hypotensive resulting in increased mortality and hospital admission. Age Ageing 45:783-788

Hintergrund. Viele ältere Patienten werden antihypertensiv behandelt. Unklar sind der Zielblutdruck einer antihypertensiven Therapie sowie die Häufigkeit niedriger Blutdruckwerte bei älteren $\mathrm{Pa}$ tienten.

Studie. In die retrospektive Beobachtungsstudie wurden ambulante Patienten jenseits des 70. Lebensjahres aufgenommen. Medizinische Ausschlusskriterien bestanden nicht. Insgesamt wurden 11.167 Patienten analysiert, 57 \% von ihnen waren Frauen.

Ergebnisse. Bei 1287 Patienten (11,6\%) fand sich ein systolischer Blutdruck von weniger als $120 \mathrm{~mm} \mathrm{Hg}$; 844 dieser Patienten erhielten eine antihypertensive Therapie, oftmals sogar eine Kombinationstherapie, in Einzelfällen mit 4 und mehr Präparaten. Niedriger als $110 \mathrm{~mm} \mathrm{Hg}$ war der systolische Blutdruck bei 474 Patienten (313 unter antihypertensiver Therapie). Bei $128 \mathrm{~Pa}$ tienten fand sich sogar ein Blutdruck von weniger als $100 \mathrm{~mm} \mathrm{Hg}$ (davon 89 unter antihypertensiver Therapie). Ein Blutdruck von weniger als $120 \mathrm{~mm} \mathrm{Hg}$ korrelierte mit erhöhter Mortalität, mit akutem Nierenversagen und mit einer höheren Zahl von Krankenhausaufnahmen.
Diskussion. Trotz methodischer Probleme belegt die Untersuchung einen klaren Zusammenhang zwischen niedrigem systolischen Blutdruck und erhöhter Sterblichkeit. Das Studiendesign lässt allerdings keine Rückschlüsse auf die Ursache des niedrigen Blutdrucks zu. Außerdem sind keine Aussagen möglich, ob beispielsweise antihypertensiv wirkende Medikamente wegen einer Herzinsuffizienz mit eingeschränkter systolischer Funktion gegeben wurden.

Fazit für die Praxis. Gerade im höheren Lebensalter erfordert eine antihypertensive Therapie eine engmaschige Überwachung. Sinkt der systolische Druck auf $120 \mathrm{~mm} \mathrm{Hg}$ oder darunter, so ist sorgfältig zu prüfen, ob die erhöhten Gefahren eines niedrigen Blutdrucks den erwarteten Nutzen der medikamentösen Therapie rechtfertigen. In den meisten Fällen wird eine Reduktion der Antihypertensiva angezeigt sein.

\section{Korrespondenzadresse}

PD Dr. R. Püllen

Agaplesion Frankfurter Diakonie-Kliniken Holzhausenstr. 72-92, 60322 Frankfurt a. M., Deutschland

rupert.puellen@fdk.info

Interessenkonflikt. R. Püllen gibt an, dass kein Interessenkonflikt besteht. 